\title{
Deformation effect on total reaction cross sections for neutron-rich Ne-isotopes
}

\author{
Kosho Minomo, ${ }^{1}$ Takenori Sumi, ${ }^{1}$ Masaaki Kimura, ${ }^{2}$ Kazuyuki Ogata, ${ }^{1}$ Yoshifumi R. Shimizu, ${ }^{1}$ and Masanobu Yahiro ${ }^{1}$ \\ ${ }^{1}$ Department of Physics, Kyushu University, Fukuoka 812-8581, Japan \\ ${ }^{2}$ Creative Research Institution (CRIS), Hokkaido University, Sapporo 001-0021, Japan
}

(Dated: November 8, 2018)

\begin{abstract}
Isotope-dependence of measured reaction cross sections in scattering of ${ }^{28-32} \mathrm{Ne}$ isotopes from ${ }^{12} \mathrm{C}$ target at $240 \mathrm{MeV} /$ nucleon is analyzed by the double-folding model with the Melbourne $g$-matrix. The density of projectile is calculated by the mean-field model with the deformed Wood-Saxon potential. The deformation is evaluated by the antisymmetrized molecular dynamics. The deformation of projectile enhances calculated reaction cross sections to the measured values.
\end{abstract}

PACS numbers: 21.10.Gv, 21.60.Cs, 21.60.Ev, 24.10.Ht, 25.60.Dz

\section{INTRODUCTION}

Exploring unstable nuclei is one of the most important subjects in nuclear physics. Actually, it was reported that unstable nuclei have exotic properties such as the halo structure [1-3] and the loss of magicity for nuclei in the so-called "Island of inversion". The term "Island of inversion" was first introduced by Warburton [4] to the region of unstable nuclei from ${ }^{30} \mathrm{Ne}$ to ${ }^{34} \mathrm{Mg}$. In the region, the low excitation energies and the large $B(E 2)$ values of the first excited states suggest strong deformations [5-9], which indicates that the $N=20$ magic number is no longer valid. These novel quantum properties have inspired extensive experimental and theoretical studies.

Important experimental tools for exploring unstable nuclei are the reaction cross section $\sigma_{\mathrm{R}}$ or the interaction cross section $\sigma_{\mathrm{I}}$ and the nucleon-removal cross section $\sigma_{-n}$ with radioactive beams $[1-3,10]$; for the scattering of unstable nuclei, $\sigma_{\mathrm{I}}$ agrees with $\sigma_{\mathrm{R}}$ in general, since projectile excitations to its discrete excited states do not exist. Very recently, $\sigma_{\mathrm{I}}$ was measured by Takechi et al. [11] for ${ }^{28-32} \mathrm{Ne}$ located near or in "Island of inversion". Furthermore, a halo structure of ${ }^{31} \mathrm{Ne}$ was reported by the experiment on the one-neutron removal reaction [12]. This is the heaviest halo nucleus in the present stage suggested experimentally and also reside within the region of "Island of inversion".

As a useful theoretical tool of analyzing $\sigma_{\mathrm{R}}$, we can consider the microscopic optical potential constructed by the double-folding model (DFM) with the $g$-matrix effective nucleon-nucleon $(\mathrm{NN})$ interaction [13-21], when the projectile breakup is weak. For the nucleon-nucleus scattering, the single-folding model with the $g$-matrix well reproduce the data on $\sigma_{\mathrm{R}}$ and the elastic-scattering cross section systematically [13]. For the ${ }^{31} \mathrm{Ne}$ scattering from ${ }^{12} \mathrm{C}$ at $240 \mathrm{MeV}$ /nucleon, the breakup cross section is at most $1.5 \%$ of $\sigma_{\mathrm{R}}$ [22]. Hence, DFM is applicable also for analyses of measured isotope-dependence of $\sigma_{\mathrm{R}}$ in the scattering of ${ }^{28-32} \mathrm{Ne}$ from ${ }^{12} \mathrm{C}$ target at $240 \mathrm{MeV} /$ nucleon [11].

In DFM, the $g$-matrix is folded with the projectile and target densities. If the projectile deforms, the density profile changes; the surface diffuseness increases because of the elongation. This gives rise to the effective growth of the rootmean-square (RMS) radius and eventually the increase of $\sigma_{R}$. Therefore, the amount of deformation is important. Nuclei in the island of inversion are spherical or only weakly deformed in the Skyrme and/or Gogny HF (HFB) calculations; see, e.g., Refs. [23, 24]. It is even pointed out that the observed large $B\left(E 2 ; 2^{+} \rightarrow 0^{+}\right)$values can be understood as a large amplitude vibration around the spherical shape [25]. In such a situation, the additional correlations by the angular momentum projection (AMP) often leads to possible deformed shapes; see Ref. [26] for Ne isotopes.

Recently a systematic investigation employing the antisymmetrized molecular dynamics (AMD) with the Gogny D1S interaction has been performed for both even and odd $N$ nuclei in the island of inversion [27]. The AMD (with AMP performed) gives rather large deformations, which is consistent with the AMP-HFB calculations [24, 26]. A consistent picture of even and odd isotopes has been obtained, where the $n$-particle $m$-hole excitations of the Nilsson orbits play important roles to determine deformed configurations. Although it is difficult to distinguish the dynamic shape-fluctuation and static deformation in these light mass nuclei, one may use the deformed shape suggested by the AMD calculation to see its effect on $\sigma_{\mathrm{R}}$.

In this paper, we analyze the measured isotope-dependence of $\sigma_{\mathrm{R}}$ in scattering of ${ }^{28-32} \mathrm{Ne}$ isotopes from ${ }^{12} \mathrm{C}$ target at $240 \mathrm{MeV} /$ nucleon, using DFM with the Melbourne $g$ matrix [13] and the deformed projectile density suggested by the AMD calculation.

\section{THEORETICAL FRAMEWORK}

A microscopic optical potential $U$ between a projectile (P) and a target (T) is constructed with DFM. The direct and exchange parts, $U_{\mathrm{D}}$ and $U_{\mathrm{EX}}$, are obtained by [28, 29]

$$
\begin{aligned}
U_{\mathrm{D}}(\boldsymbol{R})= & \int \rho_{\mathrm{P}}\left(\boldsymbol{r}_{\mathrm{P}}\right) \rho_{\mathrm{T}}\left(\boldsymbol{r}_{\mathrm{T}}\right) v_{\mathrm{D}}(\rho, \boldsymbol{s}) d \boldsymbol{r}_{\mathrm{P}} d \boldsymbol{r}_{\mathrm{T}}, \\
U_{\mathrm{EX}}(\boldsymbol{R})= & \int \rho_{\mathrm{P}}\left(\boldsymbol{r}_{\mathrm{P}}, \boldsymbol{r}_{\mathrm{P}}+\boldsymbol{s}\right) \rho_{\mathrm{T}}\left(\boldsymbol{r}_{\mathrm{T}}, \boldsymbol{r}_{\mathrm{T}}-\boldsymbol{s}\right) \\
& \times v_{\mathrm{EX}}(\rho, \boldsymbol{s}) \exp [i \boldsymbol{K}(\boldsymbol{R}) \cdot \boldsymbol{s} / M] d \boldsymbol{r}_{\mathrm{P}} d \boldsymbol{r}_{\mathrm{T}},
\end{aligned}
$$

where $s=\boldsymbol{r}_{\mathrm{P}}-\boldsymbol{r}_{\mathrm{T}}+\boldsymbol{R}$ for a position vector $\boldsymbol{R}$ of $\mathrm{P}$ from T. The original form of $U_{\mathrm{EX}}$ is a non-local function of $\boldsymbol{R}$, but it has been localized in Eq. (2) with the local semi-classical approximation [14], where $\hbar \boldsymbol{K}(\boldsymbol{R})$ is the local momentum 
of the scattering considered and $M=A_{\mathrm{P}} A_{\mathrm{T}} /\left(A_{\mathrm{P}}+A_{\mathrm{T}}\right)$ for the mass number $A_{\mathrm{P}}\left(A_{\mathrm{T}}\right)$ of $\mathrm{P}(\mathrm{T})$. The validity of this localization is shown in Ref. [30]. Here, the effective NN interactions, $v_{\mathrm{D}}$ and $v_{\mathrm{EX}}$, are assumed to depend on the local density

$$
\rho=\rho_{\mathrm{P}}\left(\boldsymbol{r}_{\mathrm{P}}+\boldsymbol{s} / 2\right)+\rho_{\mathrm{T}}\left(\boldsymbol{r}_{\mathrm{T}}-\boldsymbol{s} / 2\right)
$$

at the midpoint of the interacting nucleon pair.

The microscopic potential $U$ is not spherical, if one or both of the densities $\rho_{\mathrm{P}}$ and $\rho_{\mathrm{T}}$ are non-spherical. As shown in Ref. [17], however, the effect is found to be negligible for heavy-ion scattering. For intermediate incident energies of our interest, this can be understood with reasonable approximations. For such energies, the rotational motion of deformed $\mathrm{P}$ (rotor) is negligible compared with the center-of-mass motion of $\mathrm{P}$. Hence, the adiabatic approximation is applicable for the rotational motion. Under the approximation, $\mathrm{P}$ is scattered by $U$ depending on not only $\boldsymbol{R}$ but also $\Omega$ the direction (Euler angles) of the symmetry axis of rotor in the space-fixed frame. Using the eikonal approximation for the center-of-mass motion of $\mathrm{P}$, one can obtain

$$
\sigma_{\mathrm{R}}=\int d \boldsymbol{b}\left(1-|S|^{2}\right)
$$

with

$$
S=\int \frac{d \Omega}{8 \pi^{2}} \exp \left[-\frac{i}{\hbar v} \int_{-\infty}^{\infty} d z U(\boldsymbol{R}, \Omega)\right]
$$

for $\boldsymbol{R}=(\boldsymbol{b}, z)$ and the velocity $v$ of P. Here, we have assumed that the ground state of $\mathrm{P}$ is a $0^{+}$state for simplicity. The $S$-matrix element $S$ includes back-coupling effects of the rotational excitations on the elastic scattering. Separating $U$ into the spherical and non-spherical parts, $U_{0}$ and $\Delta U$, we can get

$$
S=S_{0}+S_{0} \int \frac{d \Omega}{8 \pi^{2}}\left(\frac{\delta^{2}}{2}+\cdots\right)
$$

with

$$
\begin{aligned}
S_{0} & =\exp \left[-\frac{i}{\hbar v} \int_{-\infty}^{\infty} d z U_{0}(R)\right] \\
\delta & =-\frac{i}{\hbar v} \int_{-\infty}^{\infty} d z \Delta U(\boldsymbol{R}, \Omega)
\end{aligned}
$$

The non-spherical correction to the spherical part $S_{0}$ starts with $\delta^{2}$, since the correction of order $\delta$ vanishes because of the angle average. The leading-order correction is significant only at large $b$, since $S_{0}$ vanishes at small $b$ as a result of the strong absorption. For large $b, \delta$ keeps small because $v$ is large and the range of the $z$ integration is small. Actually, we confirmed through numerical calculations that the leadingorder correction to $\sigma_{\mathrm{R}}$ is $0.01 \%$ for the ${ }^{30} \mathrm{Ne}+{ }^{12} \mathrm{C}$ scattering at $240 \mathrm{MeV} /$ nucleon; note that the error of the eikonal approximation is less than $1 \%$ for this scattering. Also for the case that the spin of $\mathrm{P}$ in its ground state is non-zero, it is possible to prove that the leading-order correction to $\sigma_{\mathrm{R}}$ is of order $\delta^{2}$.
Thus, the effect of $\Delta U$ on $\sigma_{\mathrm{R}}$ is negligible. Therefore, only the spherical part of the density is taken in this paper. Detailed discussion on this non-spherical effect will be made in the forthcoming paper.

As for $\rho_{\mathrm{T}}$, we use the phenomenological ${ }^{12} \mathrm{C}$-density deduced from the electron scattering [31] by unfolding the finitesize effect of the proton charge in the standard manner [32]. Meanwhile, $\rho_{\mathrm{P}}$ is calculated by the mean-field model with a given average potential or with the self-consistently determined potential by the Hartree-Fock (HF) method. No effect of pairing is included for simplicity. The Ne isotopes (projectiles) under discussions are supposed to be in the island of inversion (or at its boundary), and expected to be strongly deformed. In order to investigate the effect of deformation, we take a deformed Woods-Saxon (WS) potential [33], in which the axially deformed surface $\Sigma(\boldsymbol{\beta})$ is specified by the radius,

$$
R(\theta ; \boldsymbol{\beta})=R_{0} c_{v}(\boldsymbol{\beta})\left[1+\sum_{\lambda} \beta_{\lambda} Y_{\lambda 0}(\theta)\right],
$$

with the deformation parameters $\boldsymbol{\beta} \equiv\left\{\beta_{\lambda}\right\}$ and a volume conserving factor $c_{v}(\boldsymbol{\beta})$. The potential value is determined by replacing the quantity $\left(r-R_{0}\right)$ in a spherical potential to the distance from the surface $\Sigma(\boldsymbol{\beta})$ (with minus sign if the point is inside it). The Coulomb potential created by charge $(Z-1) e$ distributed uniformly inside the surface $\Sigma(\boldsymbol{\beta})$ in Eq. (9) is included for protons. The single-particle eigenstates are calculated by the (cylindrical) harmonic oscillator basis expansion. More than twenty oscillator shells are included and the convergence of the result is carefully checked to obtain reliable density distributions. The nucleon density is obtained by summing up the contributions of occupied Nilsson levels. The density distribution thus calculated $\rho_{\mathrm{P}}^{\text {(in) }}(r, \theta)$ is the one in the intrinsic (body-fixed) frame, and depends on the polar angle $\theta$ from the symmetry axis. As mentioned above, the density in the laboratory frame used in DFM (Eqs. (1) and (2)) is obtained by the angle average:

$$
\rho_{\mathrm{P}}(r)=\frac{1}{2} \int_{0}^{\pi} \rho_{\mathrm{P}}^{(\mathrm{in})}(r, \theta) \sin \theta d \theta .
$$

We have checked that the angle-averaged density agrees with high accuracy with the density calculated by the angular momentum projection from the Slater determinantial wave function composed of the occupied WS orbits.

No center of mass (CM) correction is included for the calculation of the density. We have checked by the spherical Gogny HF calculation that the CM correction (including the two-body contributions) to the RMS radius is about $1 \%$ reduction for all the isotopes. The amount of reduction is smaller than the enhancement caused by the deformation effect, but is non negligible; we will return to this point latter.

\section{RESULTS}

We test the accuracy of DFM with the Melbourne $g$-matrix for ${ }^{12} \mathrm{C}+{ }^{12} \mathrm{C}$ scattering at $250.8 \mathrm{MeV} /$ nucleon. As shown in Table I $\sigma_{\mathrm{R}}$ calculated with the Melbourne $g$-matrix is consistent with the experimental data; more precisely, the latter 
TABLE I: Reaction cross sections for ${ }^{12} \mathrm{C}+{ }^{12} \mathrm{C}$ scattering at $250.8 \mathrm{MeV} /$ nucleon for two types of effective nucleon-nucleon interactions. The cross sections are presented in units of mb.

\begin{tabular}{ccc}
\hline Exp. [34] & Love-Franey [35] & Melbourne- $g$ [13] \\
\hline $782.0 \pm 10$ & 918 & 796 \\
\hline
\end{tabular}

is slightly smaller than the former by the factor $F=0.982$. The table also shows the result of the Love-Franey $t$-matrix nucleon-nucleon interaction in which the nuclear medium effect is not included. The difference between the two theoretical results is about $122 \mathrm{mb}$ that corresponds to $16 \%$ of the experimental data. Thus, the medium effect is important at this incident energy. For the ${ }^{27} \mathrm{Al}+{ }^{12} \mathrm{C}$ scattering at $250.7 \mathrm{MeV} /$ nucleon, $\sigma_{\mathrm{R}}$ calculated with the phenomenological ${ }^{27} \mathrm{Al}$ density [31] and the normalization factor $F$ is $1164 \mathrm{mb}$, while the experimental value is $1159 \pm 14 \mathrm{mb}$ [34]. The normalization procedure thus justified is applied for the ${ }^{28-32} \mathrm{Ne}+{ }^{12} \mathrm{C}$ scattering at $240 \mathrm{MeV} /$ nucleon analyzed below.

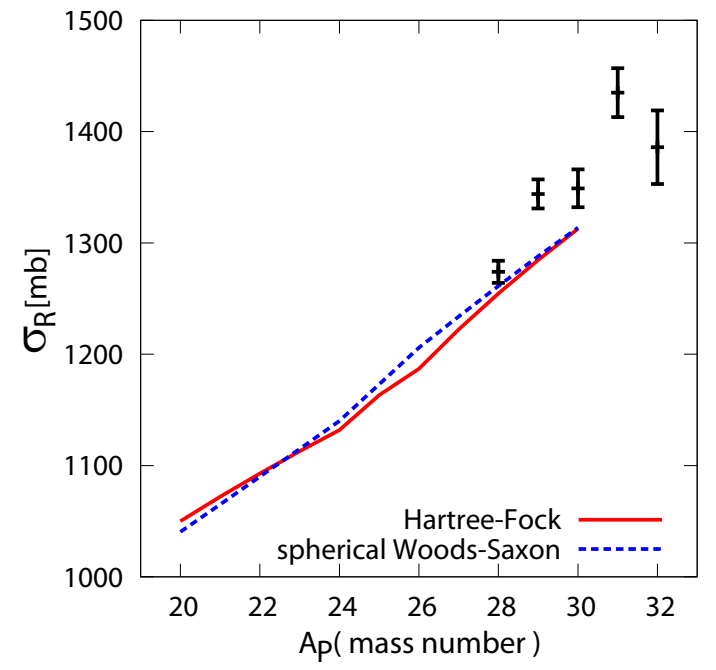

FIG. 1: (Color online) Reaction cross sections for scattering of $\mathrm{Ne}$ isotopes from ${ }^{12} \mathrm{C}$ at $240 \mathrm{MeV} /$ nucleon. The results obtained with the calculated density with the Woods-Saxon potential are denoted by dashed line, and those with the Gogny HF by solid line. The spherical shape is imposed. The nuclei with $A>30$ are unbound. The experimental data are taken from Ref. [11].

As for the parameter set of the WS potential, i.e., the depth, radius and the diffuseness of the central as well as the spinorbit potentials, we employ the one provided recently by R. Wyss [36]; see Table I of Ref. [37] for the actual values of parameters. This set is intended to reproduce the spectroscopic properties of high-spin states from light to heavy deformed nuclei, e.g., the quadrupole moments and the moments of inertia, and at the same time the RMS radii crucial for the
TABLE II: Deformation parameter $\beta_{2}$ used in the calculation of density of $\mathrm{Ne}$ isotope (those with higher multipoles $\lambda>2$ are not included).

\begin{tabular}{cccccc}
\hline nuclide & ${ }^{28} \mathrm{Ne}$ & ${ }^{29} \mathrm{Ne}$ & ${ }^{30} \mathrm{Ne}$ & ${ }^{31} \mathrm{Ne}$ & ${ }^{32} \mathrm{Ne}$ \\
\hline$\beta_{2}$ & -0.291 & 0.445 & 0.400 & 0.422 & 0.335 \\
\hline
\end{tabular}

present analysis. In order to check that the present WS potential gives reasonable results, we compare in Fig. 1 the reaction cross sections calculated by using two densities; one obtained by the Gogny D1S HF calculation and another with the WS potential: The spherical shape is imposed with the filling approximation in this calculation. A good agreement shown in the figure indicates that the density distributions in the two models are similar, which is also confirmed by the calculated RMS radii (see Fig. 3).

The reaction cross section is sensitive to the amount of deformation. We then employ the deformed shapes suggested by the AMD calculation to see the effect on $\sigma_{\mathrm{R}}$. As a simple estimate we only include the $Y_{20}$ deformation in Eq. (9) and the deformation parameter $\beta_{2}$ in each isotope is determined to reproduce the calculated ratio of RMS radii along the long and short axes by AMD; the resultant values used in the following analyses are given in Table II With these $\beta_{2}$ values, the Nilsson orbits of the last-odd-neutron in ${ }^{29} \mathrm{Ne}$ and ${ }^{31} \mathrm{Ne}$ are [200]1/2 and [321]3/2, respectively, in accordance with the AMD calculation. Note that the nucleus ${ }^{28} \mathrm{Ne}$ is at the boundary of the "Island of inversion", and AMD predicts strong mixing between the states with oblate and prolate deformation. In the present calculation, we have employed the $\beta_{2}$ value of the oblate minimum, which is the main component.

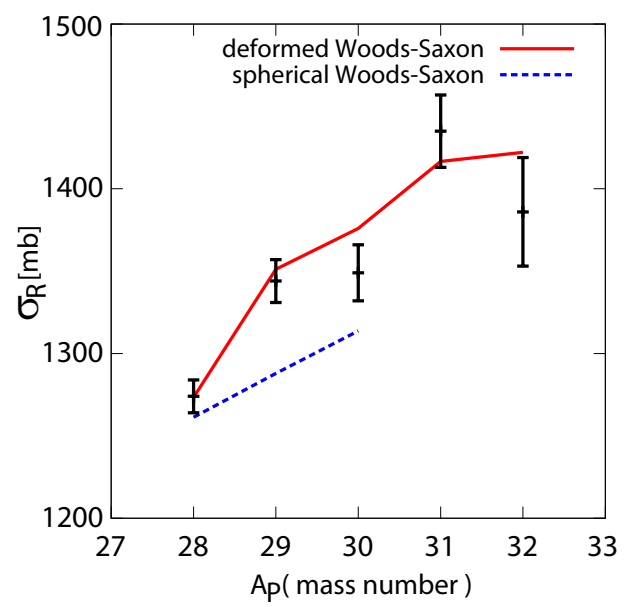

FIG. 2: (Color online) Reaction cross sections for scattering of $\mathrm{Ne}$ isotopes from ${ }^{12} \mathrm{C}$ at $240 \mathrm{MeV} /$ nucleon. The dashed and solid lines represent results of the spherical and deformed WS potentials, respectively. The experimental data are taken from Ref. [11].

The results of $\sigma_{\mathrm{R}}$ including the effect of quadrupole defor- 
mation (see Table $\amalg$ are shown in Fig. 2. Compared to the results with the density of the spherical cases, the effect of deformation increases the cross section considerably. The enhancement makes the calculated cross sections almost consistent with the measured cross sections for ${ }^{28-32} \mathrm{Ne}$, although the difference of $\sigma_{\mathrm{R}}$ between ${ }^{30} \mathrm{Ne}$ and ${ }^{31} \mathrm{Ne}$ is small in the model calculation compared with the difference deduced from the measured cross sections. We will return to this point later.

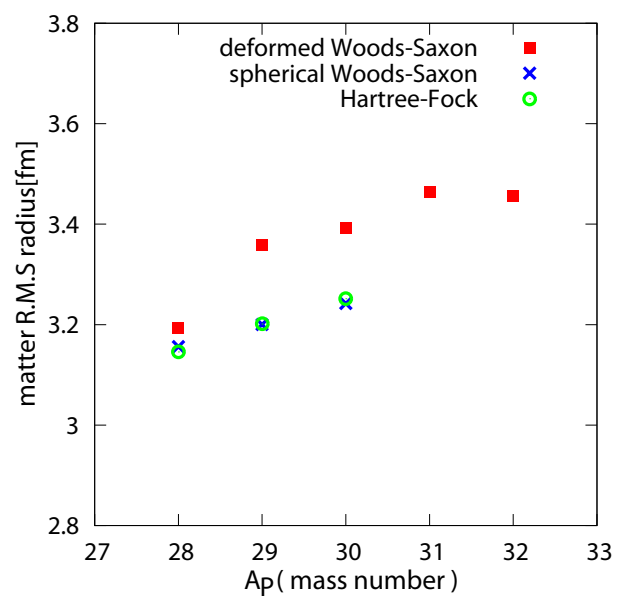

FIG. 3: (Color online) Matter RMS radii for Ne isotopes for the spherical WS potential (crosses), the deformed WS potential (squares) and the Gogny HF (circles).

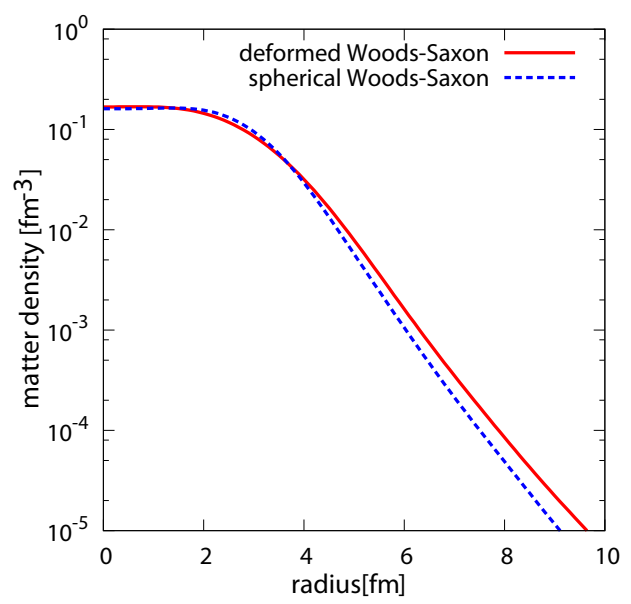

FIG. 4: (Color online) The radial dependence of the matter density for ${ }^{29} \mathrm{Ne}$. The dashed and solid lines show results of the spherical and deformed WS potentials, respectively.

The increase of $\sigma_{\mathrm{R}}$ caused by the deformation can be rather nicely understood if one looks into the (matter) RMS radii $\left\langle r^{2}\right\rangle_{\mathrm{P}}^{1 / 2}$ shown in Fig. 3. They are calculated by using the projectile density $\rho_{\mathrm{P}}$ based on the spherical and deformed WS potentials. The increase of $\left\langle r^{2}\right\rangle_{\mathrm{P}}^{1 / 2}$ in Fig. 3 nicely corresponds to that of $\sigma_{\mathrm{R}}$ in Fig. 2. which is reasonable because of a simple estimate,

$$
\sigma_{\mathrm{R}} \approx \pi\left[\left\langle r^{2}\right\rangle_{\mathrm{P}}^{1 / 2}+\left\langle r^{2}\right\rangle_{\mathrm{T}}^{1 / 2}\right]^{2}
$$

where $\left\langle r^{2}\right\rangle_{\mathrm{T}}^{1 / 2}$ is the RMS radius for target. Note that the amount of increase of the RMS radii from the spherical shape, which is roughly proportional to $\beta_{2}^{2}$, is only $4-6 \%$ : It is surprising that such a small effect is detectable in experimental data. The present analysis clearly tells us that $\sigma_{\mathrm{R}}$ reflects very precise information, and its measurement is extremely useful to study the nuclear structure of unstable nuclei. The radial dependence of the matter density is plotted in Fig. 4. The deformed WS density (solid curve) is enhanced by the deformation effect from the spherical WS density (dashed curve) at $r \gtrsim 4 \mathrm{fm}$. The enhancement of the reaction cross section is caused by that of the density in this tail region. This is the main reason why we do not directly use the calculated AMD density, which decreases more rapidly in the tail region because of the usage of one-range gaussian wave functions.

\section{DISCUSSIONS}

The enhancement of the reaction cross sections caused by the deformation effect is conspicuous as shown in Fig. 2. The enhancement makes the calculated cross sections almost consistent with the observed ones for ${ }^{28-32} \mathrm{Ne}$; more precisely, the calculated cross sections slightly overshoot the data for ${ }^{30,32} \mathrm{Ne}$, but slightly undershoot the data for ${ }^{31} \mathrm{Ne}$. In the spherical HF calculation, the CM correction to the RMS radii yields $1 \%$ reduction. This leads to $1.1 \%$ reduction of $\sigma_{\mathrm{R}}$ through relation (11). It is very likely that the deformed WS model has almost a same amount of $\sigma_{\mathrm{R}}$ reduction. After this reduction, the calculated cross sections agrees with the data for ${ }^{28-30,32} \mathrm{Ne}$, but underestimates the data by $32 \pm 22 \mathrm{mb}$ for ${ }^{31} \mathrm{Ne}$. Thus, the theoretical results are consistent with the data for ${ }^{30} \mathrm{Ne}$ but not for ${ }^{31} \mathrm{Ne}$, because the difference of $\sigma_{\mathrm{R}}$ between the two nuclei is smaller in the model calculation than in the data.

The difference of $\sigma_{\mathrm{R}}$ between ${ }^{31} \mathrm{Ne}$ and ${ }^{30} \mathrm{Ne}$ corresponds to the one-neutron removal cross section of ${ }^{31} \mathrm{Ne}$, if the breakup cross section of ${ }^{31} \mathrm{Ne}$ is negligible [22]. The difference between the observed reaction cross sections is $86 \mathrm{mb}$, while the direct measurement on the one-neutron removal cross section yields $79 \mathrm{mb}$ [12]. Thus, the two experimental data are consistent with each other, indicating that the breakup cross section is small. Meanwhile, the difference of the calculated reaction cross sections between the two nuclei is $41 \mathrm{mb}$ and smaller than the experimental results.

As for ${ }^{31} \mathrm{Ne}$, the single-particle energies of the last neutron are about $-2 \mathrm{MeV}$ in the present deformed WS potential with $\beta_{2}$ value given in Table II. The underestimation of the present value for ${ }^{31} \mathrm{Ne}$ may mean that either the depth of the present WS potential is too deep or $\beta_{2}$ is too small. For example, compared with the WS potential in Ref. [38], the binding energies of relevant Nilsson orbits are about $2 \mathrm{MeV}$ larger in the present case, though the Nilsson diagrams are very similar to 
each other. It turns out that we can obtain good agreements of $\sigma_{\mathrm{R}}$ for ${ }^{31} \mathrm{Ne}$ either by shallowing the potential depth by factor 0.943 or by increasing the deformation up to $\beta_{2}=0.590$.

In the case of ${ }^{31} \mathrm{Ne}$, its spin-parity and neutron configuration are still under debate. Our prediction of the lastodd-neutron orbit is [321]3/2 with the single-particle energy $-1.947 \mathrm{MeV}$. The energy increases to $-0.974 \mathrm{MeV}$ when the potential is reduced by a factor 0.943 to account for the observed central value of $\sigma_{\mathrm{R}}$, while the last-odd-neutron orbit changes to $[200] 1 / 2$ and the energy decreases to $-2.803 \mathrm{MeV}$ when $\beta_{2}$ is increased to 0.590 . The measured separation energy of ${ }^{31} \mathrm{Ne}, 0.29 \pm 1.64 \mathrm{MeV}$ [39], is more consistent with the single-particle energy of the shallower potential rather than that of larger $\beta_{2}$.

It should be mentioned that the present calculation of $\sigma_{R}$ is not sensitive to the isovector properties, e.g., the neutron skin. Although the matter radii calculated with the present WS and with the Gogny D1S HF (imposing the spherical shape) perfectly agree and so do the reaction cross sections (see Fig.1, the skin thicknesses in the two calculations are rather different: e.g., $\left\langle r^{2}\right\rangle_{n}^{1 / 2}-\left\langle r^{2}\right\rangle_{p}^{1 / 2} \approx 0.67$ and $0.41 \mathrm{fm}$ with the WS and the Gogny HF, respectively, in ${ }^{30} \mathrm{Ne}$. Additional information is necessary to probe the property like the skin thickness.

\section{SUMMARY}

Isotope-dependence of measured reaction cross sections in scattering of ${ }^{28-32} \mathrm{Ne}$ isotopes from ${ }^{12} \mathrm{C}$ target at
$240 \mathrm{MeV} /$ nucleon is analyzed by the double-folding model with the Melbourne $g$-matrix. The density of projectile is calculated by the mean-field model with the deformed WoodSaxon potential. The deformation is evaluated by the antisymmetrized molecular dynamics. The deformation of projectile enhances calculated reaction cross sections to the measured values. The increase of the RMS radii by the deformation is only $4-6 \%$, but it is quite important that such a small effect is detectable in the experimental data. Owing to this effect, the calculated reaction cross sections reproduce the data for ${ }^{28-30,32} \mathrm{Ne}$. For ${ }^{31} \mathrm{Ne}$, however, the present results still underestimate the measured cross sections. The underestimation may suggest that the extra weak-binding effect for neutrons plays an important role particularly for ${ }^{31} \mathrm{Ne}$.

\section{Acknowledgements}

The authors thank M. Takechi for providing the numerical data and H. Sakurai for useful discussions. This work is supported in part by Grant-in-Aid for Scientific Research (C) No. 22540285 and 22740169 from Japan Society for the Promotion of Science. The numerical calculations of this work were performed on the computing system in Research Institute for Information Technology of Kyushu University.
[1] I. Tanihata, et al., Phys. Lett. B 289, 261 (1992). I. Tanihata, J. Phys. G 22, 157 (1996).

[2] A. S. Jensen, et al., Rev. Mod. Phys. 76, 215 (2004).

[3] B. Jonson, Phys. Rep. 389, 1 (2004).

[4] E. K. Warburton, J. A. Becker, and B. A. Brown, Phys. Rev. C 41, 1147 (1990).

[5] T. Motobayashi et al., Phys. Lett. B 346, 9 (1995).

[6] E. Caurier, F. Nowacki, A. Poves, J. Retamosa, Phys. Rev. C 58, 2033 (1998).

[7] Y. Utsuno, T. Otsuka, T. Mizusaki, M. Honma, Phys. Rev. C 60, 054315 (1999).

[8] H. Iwasaki et al., Phys. Lett. B 522, 227 (2001).

[9] Y. Yanagisawa et al., Phys. Lett. B 566, 84 (2003).

[10] A. Gade, et al., Phys. Rev. C 77, 044306 (2008).

[11] M. Takechi et al., Nucl. Phys. A834, 412c (2010).

[12] T. Nakamura, et al., Phys. Rev. Lett. 103, 262501 (2009).

[13] K. Amos, P. J. Dortmans, H. V. von Geramb, S. Karataglidis, and J. Raynal, Adv. Nucl. Phys. 25, 275 (2000).

[14] F.A. Brieva and J.R. Rook, Nucl. Phys. A291, 299 (1977); ibid. 291, 317 (1977); ibid. 297, 206 (1978).

[15] G. Bertsch, J. Borysowicz, M. McManus, and W.G. Love, Nucl. Phys. A284, 399(1977).

[16] J.-P. Jeukenne, A. Lejeune and C. Mahaux, Phys. Rev. C16, 80 (1977); ibid. Phys. Rep. 25, 83 (1976).

[17] G. R. Satchler, Phys. Rep. 55, 183-254 (1979).

[18] G. R. Satchler, "Direct Nuclear Reactions", Oxfrod University Press, (1983) and the references cited therein.

[19] N. Yamaguchi, S. Nagata and T. Matsuda, Prog. Theor. Phys.
70, 459 (1983); N. Yamaguchi, S. Nagata and J. Michiyama, Prog. Theor. Phys. 76, 1289 (1986).

[20] L. Rikus, K. Nakano and H. V. von Geramb, Nucl. Phys. A414, 413 (1984); L. Rikus and H.V. von Geramb, Nucl. Phys. A426, 496 (1984).

[21] T. Furumoto, Y. Sakuragi, and Y. Yamamoto, Phys. Rev. C78, 044610 (2008); ibid., C79, 011601(R) (2009); ibid., C80, 044614 (2009).

[22] M. Yahiro, K. Ogata, and K. Minomo, arXiv:1103.3976 (2011) [nucl-th].

[23] J. Terasaki, H. Flocard, P.-H. Heenen, and P. Bonche, Nucl. Phys. A 621, 371 (1997).

[24] R. Rodríguez-Guzmán, J.L. Egido, and L.M. Robledo, Nucl. Phys. A 709, 201 (2002).

[25] M. Yamagami and Nguyen Van Giai, Phys. Rev. C 69, 034301 (2004).

[26] R.R. Rodríguez-Guzmán, J.L. Egido, and L.M. Robledo, Eur. Phys. J. A 17, 37 (2003).

[27] M. Kimura and H. Horiuchi, Prog. Theor. Phys. 111, 841 (2004); M. Kimura, Phys. Rev. C 75, 041302 (2007).

[28] B. Sinha, Phys. Rep. 20, 1 (1975). B. Sinha and S. A. Moszkowski, Phys. Lett. B81, 289 (1979).

[29] T. Furumoto, Y. Sakuragi, and Y. Yamamoto, Phys. Rev. C82, 044612 (2010).

[30] K. Minomo, K. Ogata, M. Kohno, Y. R. Shimizu and M. Yahiro, J. Phys. G 37, 085011 (2010) arXiv:0911.1184 [nucl-th]].

[31] H. de Vries, C. W. de Jager, and C. de Vries, At. Data Nucl. Data Tables 36, 495 (1987). 
[32] R. P. Singhal et al., Nucl. Instr. and Meth. 148, 113(1978).

[33] S. Cwiok, J. Dudek, W. Nazarewicz, J. Skalski and T. Werner, Comp. Phys. Comm. 46, 379 (1987).

[34] M. Takechi, et al., Phys. Rev. C 79, 061601(R) (2009).

[35] W. G. Love and M. A. Franey, Phys. Rev. C24, 1073 (1981); M. A. Franey and W. G. Love, Phys. Rev. C31, 488 (1985).
[36] R. Wyss (private communication).

[37] T. Shoji and Y. R. Shimizu, Progr. Theor. Phys. 121, 319 (2009).

[38] I. Hamamoto, Phys. Rev. C 81 021304(R) (2010).

[39] B. Jurado, et al., Phys. Lett. B 649, 43 (2007). 